\title{
Wie finden wir Fehler?
}

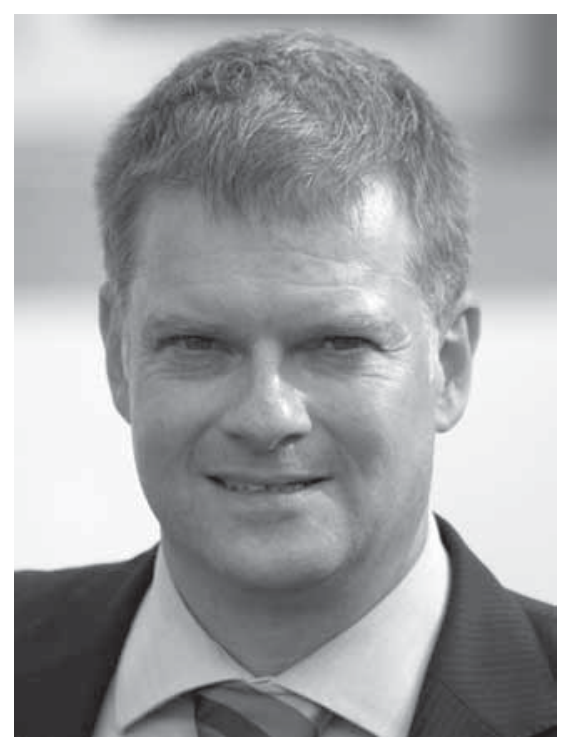

Spätestens seit Clifford Stolls „Kuckucksei“ (1989) ist die Ursache des Übels bekannt: Fehler in Programmen sind es, die es Angreifern erlauben, mithilfe passender "Exploits" Privilegien auf einem IT-System zu erlangen und es unter ihre Kontrolle zu bringen. Heute erfolgen solche Angriffe nicht mehr über langsame Modem-Verbindungen, sondern über ein breitbandiges Internet.

Seit bald 30 Jahren versuchen wir, dieses Problem in den Griff zu bekommen. Mit bescheidenem Erfolg. Und das, obwohl es riesige Fortschritte bei der Vermeidung, Identifikation und Bereinigung von fehlerhafter Software gibt: Heutige Entwicklungsumgebungen lassen zahlreiche Standard-Fehler erst gar nicht zu, CERT-Initiativen warnen systematisch vor bekannten Fehlern und systematisches Patch-Management sorgt in vielen Unternehmen für eine schnelle Verbreitung von Updates. Die Hindernisse sind jedoch bekannt: Umfang und Komplexität von Software nehmen rasant zu, ebenso wie die Zahl der Anwendungen, der Entwickler - und der Angreifer. Beschleunigt hat diese Entwicklung das Internet:Webanwendungen sind zahlreich und von überall und jedermann (fast) spurenlos angreifbar, und Schwachstellen aufWebseiten ermöglichen die Verbreitung von Schadcode, der sich auf den Systemen nichts ahnender Seitenbesucher einnistet.

Will man die Ursache sicherheitskritischer Fehler an der Wurzel bekämpfen, muss man - das ist nicht neu - für die Aufklärung und Sensibilisierung der Entwickler Sorge tragen. Ein langwieriger und oft zäher Prozess. Derweil wird weiter sicherheitskritische Software in die Welt entlassen. Denn die (heute noch überwiegend manuelle) Fehlersuche ist ein aufwändiger Prozess. Da liegt es nahe, die Suche nach sicherheitskritischen Softwarefehlern zu automatisieren. Auch das ist keine neue Idee: Statische Code-Analysen und Fuzzing, d. h. die Untersuchung des Verhaltens einer Anwendung bei zufälligen Eingaben, sind etablierte Verfahren. Sie haben jedoch vergleichsweise bescheidene Erfolgsquoten, die oft deutlich hinter denen manueller Expertenanalysen zurückbleiben.

In den vergangenen Jahren hat die Forschung in diesem Bereich jedoch erhebliche Fortschritte gemacht. Eine beeindruckende Entwicklung stellen wir in diesem Heft vor: Die von Fabian Yamaguchi und Konrad Rieck entwickelte Analyseplattform Octopus sucht mit Hilfe von Graphdatenbanken und Lernalgorithmen nach Mustern im Programmcode, die typisch für bekannte Programmierfehler sind. Die in Octopus verwendete Repräsentation des Programmcodes macht das Verfahren nahezu unabhängig von der Programmiersprache, und durch maschinelles Lernen verbessert sich die Erfolgsquote mit jeder durchgeführten Analyse. Die bisherigen Ergebnisse sind beeindruckend: Octopus fand unter anderem 18 bislang unbekannte Schwachstellen im Linux-Kern.

Derweil kämpfen wir mit einem zweiten, ähnlichen Problem: Woher wissen wir als Benutzer, dass eine Software keine Schadkomponente enthält? Insbesondere bei Apps können wir uns nicht einmal mit der Reputation des Herstellers trösten. Lädt eine App einzelne Komponenten nach oder versteckt sie Teile ihrer Funktion in verschlüsseltem Code, haben auch Virenscanner in der Regel keine Chance. Abhilfe könnte das von Siegfried Rasthofer, Steven Arzt, Eric Bodden und Marc Miltenberger entwickelte System Harvester bieten, das automatisiert Codeabschnitte einer App separiert und in einer integrierten Emulator-Umgebung kontrolliert zur Ausführung bringt. Damit lassen sich zahlreiche Verschleierungstechniken umgehen, die heute von Schadsoftware-Entwicklern eingesetzt werden. Mit Harvester könnten Apps z. B. von App-Store-Betreibern in kürzester Zeit automatisiert auf Schadsoftware analysiert werden.

Hoffentlich gelingt diesen und weiteren viel versprechenden Ansätzen möglichst bald der Schritt vom Forschungsprototyp zum erfolgreichen Produkt. Denn an der Front der Softwarefehlerbekämpfung benötigen wir solche Fortschritte dringender denn je.

Dirk Fox 